\title{
Semantic context consolidation and rule learning for optimized transport assignments in hospitals
}

\author{
F. Ongenae, P. Bonte, J. Schaballie, B. Vankeirsbilck, and F. De Turck \\ IBCN Research group, INTEC Department, Ghent University \\ iGent Building, Technology Park 15, 9052 Zwijnaarde, Belgium \\ Femke.Ongenae@intec.ugent.be
}

\begin{abstract}
The increase of ICT infrastructure in hospitals offer opportunities for cost reduction by optimizing workflows, while maintaining quality of care. This work-in-progress poster details the AORTA system, which is a semantic platform to optimize transportation task scheduling and execution in hospitals. It provides a dynamic scheduler with an upto-date view about the current context by performing semantic reasoning on the information provided by the available software tools and smart devices. Additionally, it learns semantic rules based on historical data in order to avoid future delays in transportation time.
\end{abstract}

\section{Introduction}

Healthcare is under high financial pressure and many hospitals struggle to balance budgets while maintaining quality. Hence, they are investigating ways to optimize care delivery processes. One area of interest is the organization of logistic services, which may account for more than $30 \%$ of hospital costs [4]. Logistic operations in hospitals require more resources than reasonable, both in terms of staff responsible for scheduling and dispatching the activities and in terms of executing them. Moreover, not all tasks are performed by logistic personnel. It is estimated that nurses will spend on average $10 \%$ of their time performing logistic tasks [2]. One area where there are huge opportunities in terms of gain in efficiency and cost reduction is the transport logistics of patients and equipment.

The advent of Internet of Things and intelligent decision support systems enable the design of advanced software systems to automatically assign the most suitable staff member to a transport based on all the available information about the context (e.g. location of staff and patients \& how crowded the hospital is), the staff (e.g. competences), the patient (e.g. physical condition) and the specific transportation task (e.g. pick-up location \& priority). In the AORTA project ${ }^{1}$, we aim to build such as software system to enable flexible transport task scheduling and execution through the use of smart devices, semantic context models, dynamic scheduling algorithms and self-learning models.

\section{Architecture}

The overall architecture of the designed AORTA system is visualized in Figure 1.

1 www.iminds.be/en/projects/2015/03/10/aorta 


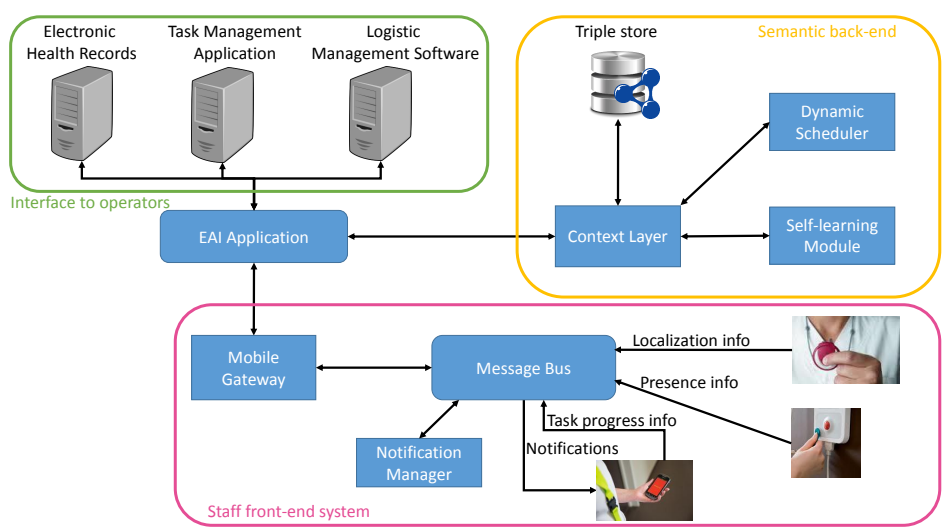

Fig. 1. The architecture of the designed AORTA system

Smart devices, wearables and sensors are introduced into the hospital environment to provide the software system with more contextual information. These devices generate dynamic data that indicate, e.g., the location of the staff or the status of a transport task. The Message Bus intercepts all these events and forwards them to interested services, which can subscribe to particular types of data through this bus. The bus also receives the notifications from the intelligent back-end software, e.g., the Dynamic Scheduler. The Notification Manager subscribes to these notifications and makes sure they are formatted in such a manner that they can be optimally shown on the device used by the staff member. The Message Bus then forwards this formatted notification to the mobile applications where they are visualized to the staff.

The Mobile Gateway forms the link between the events coming from the devices and the Enterprise Application Integration (EAI) Application. It is responsible for filtering the events generated by the mobile devices that are of interest to the back-end to schedule and execute the transportation tasks. It also makes sure that the decisions and notifications of the intelligent back-end are communicated to the front-end devices.

The EAI Application is responsible for integrating and linking the different existing software systems at the hospital. These existing software tools manage information such as the electronic health records of the patients, the transportation tasks being requested by the different hospital departments and information about the staff members and the logistics. The EAI Application is responsible for extracting the data relevant for scheduling and executing the transportation tasks from these software systems and providing it to the Context Layer.

The Context Layer consolidates and links the heterogeneous context data provided by the EAI Application by using an ontology. This semantic data is stored in a triple store. Reasoning is performed on this triple store to derive information that can be provided to the Dynamic Scheduler, e.g., the location \& current tasks of the staff, the needed transportation mode, the average walking speed within particular areas, the general commotion within the hospital and known busy periods. Moreover, based on the available context data, the reason- 
ing can also derive that an existing transportation task needs to be re-assigned, e.g., detected delays and interruptions. An overview of the task assignments by the Dynamic Scheduler is also maintained in the ontology. Based on these assignments, the context layer constructs the notifications that are forwarded to the end-users through the EAI Application. How these notifications should be sent to the staff is determined by performing semantic reasoning on the available context data about the devices and profile of the the staff, e.g., which device is a staff member currently using or his/her current location and occupation. This information can then be exploited to optimally decide which information should be contained in the notification sent to the staff member, e.g., on a mobile device an optimal route to follow to the pick-up point can be visualized and dynamically updated, while on a display on the wall only the assignment itself is shown.

The Dynamic Scheduler receives the transportation requests from the Context Layer and constructs an optimal schedule such that all the requests can be handled in a timely manner with an optimal use of resources. To achieve this optimal rostering, the scheduler will request the dynamic context information from the Context Layer, e.g., the locations, availability, competences, work load \& average walking speed of the staff, busy areas and possible causes of delay. It constantly maintains an overall optimal schedule and updates this schedules as new requests and status updates of on-going transports come in. When a staff member indicates that a transport has been finished, the Context Layer will communicate this to the Dynamic Scheduler, which will then assign a new task to this staff member based on this overall optimized schedule.

The Self-learning Module studies the scheduled assignments and accompanying executed transportation tasks to derive the reasons why some transports did not arrive on time. It extracts semantic rules, in the form of OWL axioms and adds them to the ontology. For example, the module could learn that certain transports during the visiting hour on Friday are often late and more time should be reserved for them. The incorporation of the knowledge modeled in the ontology, allows to learn more accurate rules. Furthermore, learning semantic rules allows to understand and validate the learned results. This module enables the generic system to adapt itself automatically to the workflows and particular context of the hospital in which it is deployed. Moreover, it gives insights to the staff on particular bottlenecks in their workflows or organization.

\section{Implementation}

The communication between the various components is achieved by exchanging JSON messages by using HTTP REST APIs. The Message Bus is implemented with RabbitMQ ${ }^{2}$. The EAI Application was implemented by extending the software of Xperthis ${ }^{3}$.

An ontology was constructed by extending the Task Model Ontology ${ }^{4}$, the Ambient-aware Continuous Care Ontology ${ }^{5}$ and the Amigo Location Ontology ${ }^{6}$.

\footnotetext{
2 www.rabbitmq.com/

3 www.xperthis.be/nl/integratie

5 users.intec.ugent.be/pieter.bonte/ontology/accio.htm
6 gforge.inria.fr/projects/amigo/

4 www.semanticdesktop.org/ontologies/2008/05/20/tmo/
} 
Two triple stores are currently supported, i.e., Stardog ${ }^{7} \&$ RDFox $^{8}$. Semantic reasoning is implemented by defining rules in these stores. The Context Layer communicates with the Dynamic Scheduler by translating the incoming JSON requests to SPARQL queries on the stores and forwarding the results.

The organization of transports in hospitals fits within the problem class of dynamic pick-up and delivery problems (DPDPs), which focus on goods requiring pick-up from and delivery to specific locations by a fleet of vehicles [1]. However, the constraints imposed by hospital policies and management (e.g., certain corridors that can only be used to transport goods), the relatively high request arrival rates and short transport times make it a challenging variant. In close collaboration with the hospital staff, a weighted objective function of key performance indicators, e.g., tardiness, travel time and avoiding unoccupied staff, was defined. The Dynamic Scheduler implements an optimization algorithms that iteratively tries to minimize the objective function, while upholding the defined constraints. As such, a trade-off can be made between the time available to perform the scheduling and the achieved algorithm performance.

Inductive Logic Programming was used to learn the semantic rules by employing DL-Learner [3]. As there are various reasons that cause transports to be delayed, semantic clustering is first performed on the set of late transports. Each set is then fed to DL-Learner separately and the results are merged afterward.

\section{Conclusions and next steps}

With this poster, we hope to get some initial feedback on the overall concept of using semantic technologies in order to optimize transportation task scheduling and execution within hospitals. We are currently collecting anonymized data sets from two hospitals during a period of three months, containing a realistic overview of the requested transports, their associated context and how they were scheduled and executed. This data will be used to evaluate the designed system.

Acknowledgment: This research was partly funded by the AORTA project, co-funded by the IWT, iMinds, Xperthis, Televic Healthcare, AZMM and ZNA.

\section{References}

1. G. Berbeglia, e.a.: European journal of operational research. Dynamic pickup and delivery problems 202(1), 8-15 (2010)

2. Landry, S., Philippe, R.: How logistics can service healthcare. Supply Chain Forum $5(2), 24-30(2004)$

3. Lehmann, J.: Dl-learner: Learning concepts in description logics. J. Mach. Learn. Res. 10, 2639-2642 (2009)

4. S. Hastreiter, e.a.: Benchmarking logistics services in German hospitals: A research status quo. In: ICSSSM. pp. 803-808 (2013)

7 stardog.com

8 www.cs.ox.ac.uk/isg/tools/RDFox/ 